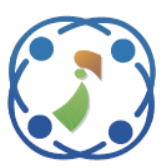

\title{
Energy Aware Optimal Cluster Head Selection Using Hybrid Algorithm for Clustering Routing in Wireless Sensor Networks
}

\author{
Rakesh Kumar Yadav ${ }^{1 *}$ \\ Rajendra Prasad Mahapatra ${ }^{1}$ \\ ${ }^{1}$ Department of Computer Science \& Engineering, SRM Institute of Science \& Technology, \\ NCR Campus Modinagar, Ghaziabad, India \\ * Corresponding author's Email: rkycse@gmail.com
}

\begin{abstract}
Clustering is one of the most significant methods for maximizing the lifetime of the network in wireless sensor networks (WSNs). The technique involves the first grouping of sensor nodes to clusters and second selecting appropriate cluster heads $(\mathrm{CHs})$ for each cluster. In fact, $\mathrm{CHs}$ gather data from corresponding nodes and transmit those aggregated data to Base Station (BS). However, the major shortcoming drawn in this technique is to choose optimal CHs. More research works have been already under the investigation on selecting the appropriate $\mathrm{CH}$ with respect to different angles. In this scenario, this paper aims to propose a new energy-aware $\mathrm{CH}$ selection by hierarchical routing in WSN via proposing a new hybrid optimization technique. More importantly, the selection of $\mathrm{CH}$ is carried out under the consideration of stabilization of energy, minimization of the distance between nodes and minimization of delay during data transmission as well. With this, a non-linear objective function is framed, which intent to optimize the $\mathrm{CH}$ in such a way that the network lifetime has to be prolonged. Here, for attaining optimal $\mathrm{CHs}$, a new hybrid algorithm is introduced that integrates the concept of Lion Algorithm (LA) and Grey Wolf Optimizer (GWO), which is named as Lion Updated GWO (LU-GWO). Finally, the performance of the proposed model is evaluated by comparing other conventional models with respect to certain performance measures. The simulation analysis shows that the proposed scheme for 50 nodes and $1000 \times 1000$ distance was $2.7 \%, 2.7 \%, 5.56 \%$, $5.56 \%$, and $5.56 \%$ better than GWO, LA, GA, PSO and ABC algorithms with respect to the alive node analysis and the overall analysis proves the superiority of proposed work.
\end{abstract}

Keywords: Wireless sensor networks, Network lifetime, Maximization, Clustering, Optimization, Hybrid.

\section{Abbreviation}

\begin{tabular}{|c|c|}
\hline$D M\left(a^{*} b\right)$ & Distance matrix \\
\hline$e_{N_{c}}$ & Euclidean distance among a CH \\
\hline$E_{e l}$ & Tlectronic energy \\
\hline$E_{T X}(N: e)$ & Potal utilized energy \\
\hline$E_{a e}$ & Energy consumed during data aggregation \\
\hline$E_{p w}$ & Required energy \\
\hline$E_{f s}$ & Energy required throughout the idle state \\
\hline$E_{1}$ & Cost of energy throughout sensing process \\
\hline$E_{S}$ & Normal node belongs to $x^{\text {th }}$ cluster \\
\hline$X_{x}$ & CH of $x^{\text {th }}$ cluster, \\
\hline$C_{x}$ & Distance between the BS and CH, \\
\hline$C_{x}-B_{s}$ & \\
\hline
\end{tabular}

\begin{tabular}{|c|c|}
\hline$C_{x}-X_{x}$ & Distance between the normal node \& CH \\
\hline$X_{x}-X_{y}$ & Distance among two normal nodes \\
\hline Len & lion's length \\
\hline$N_{x}$ and $N_{y}$ & Nodes that do not belong to $x^{\text {th }} \& y^{\text {th }}$ cluster \\
\hline$F^{\text {nd }}$ & Nomadic lion \\
\hline$F^{\text {fem }}$ & Lioness \\
\hline$F^{\text {mal }}$ & Territorial lion \\
\hline$\hat{n}$ and $\hat{m}$ & Variables \\
\hline$L a_{r}$ & Rate of laggardness \\
\hline$S t_{r}$ & Sterility rate \\
\hline$i t$ & Generation count \\
\hline$\alpha$ & Leader \\
\hline$U$ and $Y$ & Coefficient vectors, \\
\hline$F_{p}$ & Prey's position vectors \\
\hline
\end{tabular}




\begin{tabular}{|c|c|}
\hline$F$ & Position vectors of grey wolves \\
\hline$i t$ & Present iteration \\
\hline$r_{1} r_{2}$ & Random vectors that lie among $[0,1]$ \\
\hline$i t_{\max }$ & Maximum iteration. \\
\hline
\end{tabular}

\section{Introduction}

Various sensor nodes are distributed over the networks in a geographical area. The sensors that existed in the network are formed to be clustered, which saves more energy and enhances the life span of the network as well. This is actually termed to be the clustering technique in WSN. Moreover, in every cluster, one sensor node will behave as $\mathrm{CH}$ [1$3]$ and the other sensor nodes behave as member nodes. CHs includes two parameters: Initial one is the distance-based selection system, in which during the selection process, all nodes in the cluster have to be located at a specific distance from the $\mathrm{CH}$ that is nearer to it. The subsequent one is size constrained selection, in which every cluster in a network must include a restricted number of members. Accordingly, in a cluster, 3 kinds of nodes are found. The initial one is the $\mathrm{CH}[4,5]$ which makes coordination between nodes and the subsequent one is the cluster member. Third one is the cluster gateway, which links one cluster with another and passes the information among clusters [5-7]. Hierarchical based routing models were deployed for increased robustness, strong scalability and reduced data retransmission. For enhancing the life span of the system [8], hierarchical routing protocols assist in network scalability, data collection and also it minimizes the delay in data. In numerous works, it is described that the hierarchical routing especially the clustering systems creates a huge development on WSNs. Hierarchical routing is exploited for enhancing the network performance and energy utilization while the entire network sensor nodes are sending data to the BS. The core elements of the cluster based WSNs are sensor nodes, clusters, CH's, $\mathrm{BS}$ and end users. The controlling of every cluster is carried out by the $\mathrm{CH}$ [9] that has direct communication with the BS. In specific conditions, the nodes of sensor include reduced signal power and therefore it does not transfer any data. Every node in WSN passes the data to BS directly via multi-hop protocols $[10,11]$. The various hierarchical routing protocols based on clustering methods are Low-Energy Adaptive Clustering Hierarchy (LEACH) [12-14], Stable Election Protocol (SEP) [15], Power Efficient Gathering in Sensor Information System (PEGASIS) is a chain based protocol and TEEN $[16,17]$.
In WSNs, several clusters are formed due to a network partition. Each cluster has several nodes and that nodes send its information to the $\mathrm{CH}$. This $\mathrm{CH}$ gathers and aggregates the data from nodes and forward that aggregated data to sink node in clustering routing protocols. The major contribution of this paper is to present a novel technique for energy-aware optimal CHS in WSN by hierarchical routing using the hybrid optimization technique. The major goal of this paper is to optimally select the $\mathrm{CH}$ by concerning the stabilization of energy, minimization of distance among nodes and minimization of delay during data transmission in WSN. Here, by means of the above-mentioned measures, a non-linear objective function is framed, which intent to optimize the $\mathrm{CH}$ in such a way that the network lifetime has to be higher. For attaining optimal CHS, the concept of LU-GWO is introduced in this work that incorporates the concept of LA in the GWO model. Thus, optimal CHS could be efficiently determined by the adopted model. Finally, the simulation analysis shows that the, the proposed scheme for 50 nodes and $1000 \times 1000$ distance was $2.7 \%, 2.7 \%, 5.56 \%, 5.56 \%$, and $5.56 \%$ better than GWO, LA, GA, PSO and ABC algorithms with respect to the alive node analysis and the overall analysis proves the superiority of proposed work.

The arrangement of the paper is as follows. Section 2 reviews the CHS in WSN. Section 3 describes the hierarchical routing based energy aware $\mathrm{CH}$ selection in WSN and section 4 portrays the objective model for optimal CHS. Section 5 describes the optimal CHS via proposed optimization algorithm. Also, section 6 illustrates the outcomes and section 7 concludes the paper.

\section{Literature review}

In article [1], authors introduced Fuzzy Multi Cluster-Based Routing with Constant Threshold (FMCR-CT) scheme which attains a better life span and it enhances the scalability. However, it needs consideration on energy consumption. Genetic algorithm (GA) method was exploited in [2] that minimize the energy consumption and it also offers increased energy efficiency, but it requires analysis using meta-heuristic approaches. In addition, Mobile Energy Aware Cluster-Based Multi-hop (MEACBM) method was deployed in [3] that increase the throughput and it also offers more stability. Anyhow, requires analysis of the stability of nodes in every cluster. Likewise, EnergyEfficient Clustering (ENEFC) method was exploited in [4], which enhances the energy efficiency and it also offers optimal routing. However, it requires consideration on cost field. Also, decentralized 
energy efficient hierarchical cluster-based routing algorithm (DHCR) model was employed in [5], which offers enhanced stability and it offers better life span; however, node functionality has to be focused more. Mixed Integer Nonlinear Programming (MINP) was exploited in [6] that provide increased life span and it offers the better performance of network, anyhow it requires a lightweight approach for simpler implementation. Novel Energy Aware Hierarchical Cluster (NEAHC) model was implemented in [7], which offers better PDR and it also offers extended life span, but it has to concern more on the range of CH's. At last, Adjustable Range-Based Immune hierarchy clustering (ARBIC) was suggested in [8] that provides optimal connectivity and enhanced stability. However, it has to focus more on increased overhead. These limitations have to be regarded for improving the hierarchical routing based CHS in WSN systems

\section{Hierarchical energy aware $\mathrm{CH}$ selection in WSN}

\subsection{Network model}

WSN contains several sensor nodes, denoted by $\mathrm{N}_{\mathrm{N}}$, in which every sensor has its equivalent competences and are stationary. Throughout the transmission of data, a node can act as a $\mathrm{CH}$ and an active sensor. Usually, the WSN design is related to the allocation of sensors, data sensing, radio communication, topology features, and energy utilization. A sensor can be situated by random or manual mode in application areas. Here, the CHS of WSN with numerous sensors nodes and centralized BS. The gathering of a group node comprises the clustering process. It is a well-known technique to expand the lifespan of WSN. Clusters are produced by assembling the sensor nodes during the clustering process, in which a $\mathrm{CH}$ is selected, and the count of $\mathrm{CH}$ 's is indicated by $\mathrm{N}_{\mathrm{C}}$. In numerous routing protocols especially hierarchical routing, the desired CHS in terms of position and energy is considered as the main challenge. In the majority of optimization schemes, energy or distance contributes a major role, while selecting a $\mathrm{CH}$. As a result, it is essential to consider the numerous objectives to improve the node's life span. In addition, the major constraints that have to be regarded to choose the $\mathrm{CH}$ from a collection of sensor nodes are delay, energy, and distance.

\subsection{Distance model}

At first, the total selected CH's in the network transmits the message to state in which they operate as the $\mathrm{CH}$. In these situations, each sensor node evaluates the precise distance from $\mathrm{CH}$. Therefore, a node is owned by a specific cluster only, if its distance from $\mathrm{CH}$ of that cluster is minimal and, further, it transmits the message to the respective $\mathrm{CH}$. In contrast, the sensor node transmits the messages to the BS directly, if the distance among the $\mathrm{CH}$ and node is more than the distance among the BS and node. It forms a set-up for cluster formation depending on the calculation of close by distances. Therefore, the nodes could be clustered again in the network with the chosen $\mathrm{CH}$ by means of a $D M(a \times b)$ as revealed in Eq.(1), normal node and the sensor nodes are denoted by $x_{1}, x_{2}, \ldots . x_{n}$. Assume two sensor nodes $q$ (normal node) and $p$ $(\mathrm{CH})$ and their positions $y$ and $z$, correspondingly. Eq. (2) shows the formulation for measuring the Euclidean distance. As revealed in Eq. (1), the distance matrix of every element indicates the distance, which lies among the $\mathrm{CH}$ of $q^{\text {th }}$ node and $p^{\text {th }}$ node. In the matrix points, the columns having the least value have relation with the corresponding one. Consider an element $e_{N_{c 2}, x_{1}}$ that occupies the matrix's first column with reduced distance. Here, the $\mathrm{CH} N_{c 2}$ and node $x_{1}$ are linked to one another. In addition, a time slot is allotted by $N_{c}$ to each sensor nodes during data transmission. Therefore the major task of each $N_{c}$ is to assemble the transmitted data from all sensor nodes that contain the clusters. Following the attainment of data from the entire sensor nodes in the specific cluster, $N_{c}$ conveys the related data to the BS or sink. When $N_{c}$ exists at active mode, the sensor node continues in sleep mode. The operation of data transmission and reclustering is sustained from several cycles, till all the sensor nodes become inactive.

$$
\begin{gathered}
D M(a \times b)=\left[\begin{array}{l}
e_{N_{c_{c},}, x_{1}} e_{N_{c 1}, x_{2}} \ldots \ldots \ldots . . e_{N_{c l}, x_{n}} \\
e_{N_{c 2}, x_{1}} e_{N_{c 2}, x_{2}} \ldots \ldots . . . e_{N_{c 2}, x_{n}} \\
\vdots \\
\vdots \\
e_{N_{o m}, x_{1}} e_{N_{m m}, x_{2}} \ldots \ldots \ldots . . e_{N_{o n}, x_{n}}
\end{array}\right] \\
e_{p, q}=\sqrt{\left(p_{y}-q_{y}\right)^{2}+\left(p_{z}-q_{z}\right)^{2}}
\end{gathered}
$$




\subsection{Energy model}

In WSN, energy utilization remains as a major issue. Actually, the battery deployed in WSN could not be re-energized, that is, there will be no supply of power if the battery is down. Usually, additional energy is necessary to transmit the data to BS from all the sensor nodes. Generally, the network utilizes further energy as it carries out various functions such as reception, transmission, aggregation, and sensing. Therefore, the design of entire requirement of energy while transferring messages is given by Eq. (3), $E_{e l}$ depending on various constraints including filtering, digital coding, spreading, etc and $E_{T X}(N: e)$ necessary for transferring $N$ bytes of packets at distance $e$. The model for electronic energy is depicted by Eq.(4). The entire utilized energy necessary for attaining $N$ bytes of packets at distance $d$ is given by Eq. (5) and Eq. (6) shows the energy necessary for amplification. In Eq. (3), $e_{0}$ is evaluated as per Eq. (7), $E_{p w}$ and $E_{f s}$ deploying free space model. Altogether, the entire network energy could be indicated by Eq. (8). It is necessary to reduce the entire energy as in Eq. (8).

$$
\begin{aligned}
& E_{T X}(N: e)=\left\{\begin{array}{l}
E_{e l} \times N+E_{f s} \times N \times e^{2}, \text { if } e<e_{0} \\
E_{e l} \times N+E_{p w} \times N \times e^{2}, \text { if } e \geq e_{0}
\end{array}\right. \\
& E_{e l}=E_{T X}+E_{a e} \\
& E_{R X}(N: e)=E_{e l} N \\
& E_{a m}=E_{f s} e^{2} \\
& e_{0}=\sqrt{\frac{E_{f s}}{E_{p w}}} \\
& E_{\text {total }}=E_{T X}+E_{R X}+E_{1}+E_{S}
\end{aligned}
$$

\section{Objective model for optimal $\mathrm{CH}$ selection}

\subsection{Objective model}

In the proposed research work on CHS, the main objective function intends to minimize the distance among the node and the selected $\mathrm{CH}$ and it also intends to minimize the delay to transfer the data from one node to another. On the other hand, the network energy has to be higher, that is, it must utilize only a reduced amount of energy while transmitting the data. Eq. (9) reveals the objective function of adopted $\mathrm{CH}$ model, in which the value of $\eta$ has to lie in the range $0<\eta<1$. Here, $v_{m}$ and $v_{n}$ indicate the operations as denoted by Eq. (10) and Eq. (11), correspondingly. The constraints depending on delay, energy, and distance are indicated by $\sigma_{1}, \sigma_{2}$ and $\sigma_{3}$. The condition of these constrains are specified as $\sigma_{1}+\sigma_{2}+\sigma_{3}=1$. In Eq. (11), $X^{x}-B_{s}$ denotes the distance among the normal and sink node.

$$
\begin{aligned}
& H_{n}=\eta v_{n}+(1-\eta) v_{m} \\
& v_{m}=\sigma_{1} \times v_{i}^{d i s}+\sigma_{2} \times v_{i}^{e n e}+\sigma_{3} \times v_{i}^{d e l} \\
& v_{n}=\frac{1}{b} \sum_{x=1}^{b}\left\|X^{x}-B_{s}\right\|
\end{aligned}
$$

The fitness function for distance is represented by Eq. (12), in which $v_{(m)}^{d i s}$ is related with the transmission of packets from the normal node to $\mathrm{CH}$ and then from $\mathrm{CH}$ to BS. $v_{i}{ }^{\text {dis }}$ have to lie among [0, 1]. The value of $v_{i}{ }^{d i s}$ turns out to be higher, while the distance between the $\mathrm{CH}$ and normal node is high.

$$
\begin{gathered}
v_{i}^{d i s}=\frac{v_{(m)}^{d i s}}{v_{(n)}^{d i s}} \\
v_{(m)}^{d i s}=\sum_{x=1}^{N_{x}}\left[\left\|C_{x}-B_{s}\right\|+\sum_{y=1}^{N_{y}}\left\|C_{x}-X_{x}\right\|\right] \\
v_{(n)}^{d i s}=\sum_{x=1}^{N_{x}} \sum_{y=1}^{N_{y}}\left\|X_{x}-X_{y}\right\|
\end{gathered}
$$

Eq. (13), and Eq. (14) shows the formulation of $v_{(m)}^{d i s}$ and $v_{(n)}^{\text {dis }}$ correspondingly. Eq. (15) demonstrates the energy's fitness function. The value of $v_{i}^{\text {ene }}$ turns out to be high than one and the entire $\mathrm{CH}$ cumulative $v_{(m)}^{\text {ene }}$ and $v_{(n)}^{\text {ene }}$ considers the maximal energy value and the higher count of $\mathrm{CH}$. The delay's fitness function is portrayed by Eq. (15) that is directly proportional to the entire number of nodes, which are owned by the cluster. Thus, there is a reduced delay while the cluster involves only adequate count of nodes. The higher count of 
clusters is considered for reducing the delay as per Eq. (16). The denominator $N_{N}$ specifies the entire amount of nodes in WSN. Accordingly, the value $v_{i}^{d e l}$ should lie within $[0,1]$.

$$
\begin{gathered}
v_{i}^{\text {ene }}=\frac{v_{(m)}^{\text {ene }}}{v_{(n)}^{\text {ene }}} \\
v_{i}^{\text {del }}=\max \frac{\left(\| C_{x}-X_{x}\right)_{x=1}^{N_{e}}}{N_{n}}
\end{gathered}
$$

\section{Optimal CH selection via proposed optimization algorithm}

\subsection{Conventional lion optimization}

The LA [18] was established by Raja kumar that exhibits the living nature of lion species. Different from other cat species, lions continue to exist with a fascinating social system/behavior, known as pride, to make stronger their own species at all generations. LA approach includes for major steps namely, mating, generation of pride, territorial takeover and territorial defense. Here, $F$ denotes the solution vector and is addressed as $F=\left[f_{1}, f_{2}, \ldots ., f_{\hat{m}}\right]$.

\subsubsection{Pride generation}

The pride initialization is done using a $F^{\text {nd }}$, $F^{f e m}$ and $F^{m a l}$. The vector element of $F^{\text {nd }}, F^{\text {fem }}$ and $F^{m a l}$ is represented as $f_{\text {len }}^{\text {nd }}, f_{\text {len }}^{f e m}$ and $f_{\text {len }}^{m a l}$ that is regarded as the arbitrary numbers, which relies in the minimum and maximum limits while $\hat{m}>1$, where $l e n=1,2 \ldots . .$, Len. When $\hat{m}=1$ the parameters specified in Eq. (18) and (19) should be satisfied and $R\left(f_{\text {len }}\right)$ is specified in Eq. (20).

$$
\begin{gathered}
\text { Len }= \begin{cases}\hat{m} ; & \hat{m}>1 \\
\hat{n} ; & \text { otherwise }\end{cases} \\
\quad R\left(f_{\text {len }}\right) \in\left(f_{\text {len }}^{\min }, f_{\text {len }}^{\max }\right)
\end{gathered}
$$

$$
\hat{n} \% 2=0
$$

$$
R\left(f_{l e n}\right)=\sum_{l e n=1}^{\text {Len }} f_{\text {len }} 2^{\left(\frac{\text { Len }}{2}-l e n\right)}
$$

\subsubsection{Fertility evaluation}

The global or local optimum is obtained by laggards while $F^{m a l}$ and $F^{f e m}$ are saturated by fitness. In this approach, $L a_{r}$ and laggard is considered as $F^{\text {mal }}$, while $h\left(F^{m a l}\right)$ is beyond $h^{r}$ it denotes the reference fitness. The fertility of $F^{f e m}$ is denoted by $S t_{r}$ after crossover and is increased by 1 . While $S t_{r}$ is greater than tolerance $S t_{r}^{\max }$, then $F^{\text {fem }}$ update is performed according to Eq. (21). The mating will be carried out while the updated female $F^{f e m+}$ is regarded as $F^{f e m}$, because of its improvement. In Eq. (21) and Eq. (22), $f_{\text {len }}^{\text {fem+ }}$ and $f_{d}^{f e m}$ are concerned as $l e n^{\text {th }}$ and $d^{\text {th }}$ vector element of $F^{\mathrm{fem}+}$.

$$
\begin{aligned}
f_{\text {len }}^{\text {fem }+} & = \begin{cases}f_{d}^{f e m+} & \text { if len }=d \\
f_{\text {len }}^{f e m} & \text { otherwise }\end{cases} \\
f_{d}^{f e m+} & =\min \left[f_{d}^{\max }, \max \left(f_{d}^{\min }, \nabla_{d}\right)\right] \\
\nabla_{d} & =\left[f_{d}^{f e m}+\left(0.1 \breve{r}_{2}-0.05\right)\left(f_{d}^{\text {mal }}-\breve{r}_{1} f_{d}^{f e m}\right)\right]
\end{aligned}
$$

The variable $d$ is generated that lies among [1, Len ] and $\nabla$ indicates the female update process and $\breve{r}_{1} \breve{r}_{2}$ specifies the random constraints, which lies among $[0,1]$

\subsubsection{Mating}

The two foremost steps are crossover and mutation and gender-based clustering is known as the additional phase. By carrying out mutation and crossover, the cubs are created as $F^{\text {fem }}$ and $F^{m a l}$.

\subsubsection{Lion operators}

The territorial defense is represented by survival fight that generates nomad coalition and coalition updates. $F^{e-n d}$ is selected if Eq. (24) to Eq. (26) are met with.

$$
\begin{aligned}
& h\left(F^{e-n d}\right)<h\left(F^{m a l}\right) \\
& h\left(F^{e-n d}\right)<h\left(F^{m a l_{-} c u b}\right) \\
& h\left(F^{e-n d}\right)<h\left(F^{\text {fem_cub }}\right)
\end{aligned}
$$

The pride update occurs after the failure of $F^{m a l}$, while the nomad coalition update occurs only after the failure of $F^{\text {nd }}$. Territorial takeover update $F^{m a l}$ 


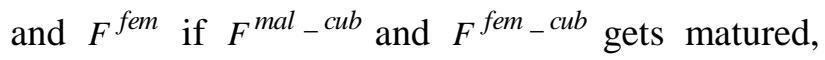
i.e. when the cub's age is greater than the maximum age for cub maturity, $A^{\max }$.

Termination: The algorithm gets terminated only if Eq. (27) or Eq. (28) are satisfied. In Eq. (27), it is initially set as 0 and raised by 1 when territorial takeover is carried out.

$$
\begin{aligned}
& i t>i t_{\max } \\
& h\left(F^{m a l}\right)-h\left(F^{o p t}\right) \leq e r_{t h}
\end{aligned}
$$

The maximal generations and threshold for error is denotes as $i t_{\max }$ and $e r_{\text {th }}$ respectively.

\subsection{Grey wolf optimization}

The mechanism of GWO [19], algorithm describes the grey wolves' hunting character and its headship hierarchy. There exist 4 types of grey wolves, such as $\alpha, \beta, \delta \quad \zeta$ that is deployed for performing the leadership nature. Penetrating, circling, and searching the food are the three foremost performances involved in hunting that developing the optimization process. The wolves $\alpha$, $\beta$ and $\delta$ are the major wolves, which focuses on the process of hunting. Among these wolves, $\alpha$ that makes decisions relating to hunting process, sleeping location, time to awake, etc. While, $\beta$ and $\delta$ holds a $2^{\text {nd }}$ and $3^{\text {rd }}$ level that aids $\alpha$ in taking decisions. In addition, the final level of wolves is concerned as $\zeta$, which concern on eating. The encircling characteristics are modeled as per Eq. (30) and Eq. (31). Eq. (33) and Eq. (32) denotes the model for $U$ and $Y$, where $\hat{a}$ is a parameter which is reduced gradually from 2 to 0 in whole iterations. The benchmark formulation $\hat{a}$ is given by Eq. (29).

$$
\begin{aligned}
& \widehat{a}_{1}=2-1 *\left(\frac{2}{i t_{\max }}\right) \\
& D=\left|Y \cdot F_{p}(i t)-F(i t)\right| \\
& F(i t+1)=F_{p}(i t)-U . D \\
& U=2 \hat{a} \cdot r_{1}-\hat{a} \\
& Y=2 r_{2}
\end{aligned}
$$

The arithmetical formula for describing the hunting character of wolves is given from Eq. (34) to Eq.
(39), where the last updated position is specified in Eq. (40), which provides the modified $F$.

$$
\begin{aligned}
& D_{\alpha}=\left|Y_{1}-F_{\alpha}-F\right| \\
& D_{\beta}=\left|Y_{2}-F_{\beta}-F\right| \\
& D_{\delta}=\left|Y_{3}-F_{\delta}-F\right| \\
& F_{1}=F_{\alpha}-U_{1} \cdot\left(D_{\alpha}\right) \\
& F_{2}=F_{\beta}-U_{2} \cdot\left(D_{\beta}\right) \\
& F_{3}=F_{\delta}-U_{3} \cdot\left(D_{\delta}\right) \\
& F(i t+1)=\frac{F_{1}+F_{2}+F_{3}}{3}
\end{aligned}
$$

\subsection{Proposed LU-GWO algorithm}

As GWO includes certain shortcomings like low

\begin{tabular}{|c|}
\hline Algorithm 1: Proposed LU-GWO Algorithm \\
\hline Parameter Initialization \\
\hline Evaluate the fitness of entire search agents \\
\hline Determine $D_{\alpha}$ using Eq. (34) \\
\hline Determine $D_{\beta}$ using Eq. (35) \\
\hline Determine $D_{\delta}$ using Eq. (22) \\
\hline Set $F_{\alpha}$ as best search agent \\
\hline Set $F_{\beta}$ as $2^{\text {nd }}$ best search agent \\
\hline Set $F_{\delta}$ as $3^{\text {rd }}$ best search agent \\
\hline While $\left(i t<i t_{\max }\right)$ \\
\hline For every wolf \\
\hline Update position as per Eq. (40) \\
\hline End for \\
\hline Update $\hat{a}, U$ and $Y$ \\
\hline
\end{tabular}
precision and slower rate of convergence, more enhancements are needed for better results. To improve the performance of conventional GWO, the proposed logic prevails over the abovementioned challenges by hybridizing GWO with LA algorithm. In the conventional GWO approach, $D_{\delta}$ is determined using Eq. (36), however, to improve the efficiency of optimal CHS, in the proposed work, $D_{\delta}$ is evaluated using the female update of LA, i.e. using Eq. (22). With the new evaluation, the updating is performed. The pseudocode for adopted LU-GWO scheme is specified by algorithm 1 and the flow chart is given in Fig. 1. 


\begin{tabular}{|l|l|}
\hline & Calculate fitness for all search agents \\
\hline & Update $F_{\alpha}, F_{\beta}$ and $F_{\delta}$ \\
\hline & it $=i t+1$ \\
\hline End while \\
\hline Return $F_{\alpha}$ \\
\hline
\end{tabular}

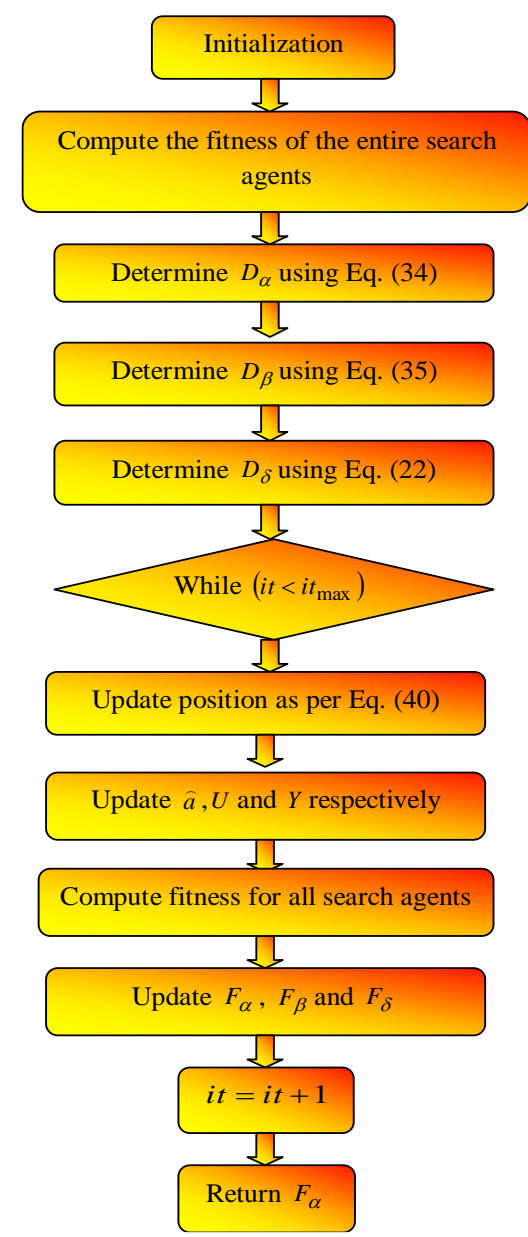

Figure. 1 Flowchart of the proposed LU-GWO model

Table 1. Simulation parameters

\begin{tabular}{|c|c|c|}
\hline S.No & Parameter & Value \\
\hline 1 & Nodes in WSN $\left(N_{N}\right)$ & $1000 \mathrm{~m} \times 1000 \mathrm{~m}$ \\
\hline 2 & Number of rounds & 0 to 100 rounds \\
\hline 3 & Initial Energy $\left(E_{i}\right)$ & 25 \\
\hline 4 & Receiving power & $0.0648 \mathrm{~nJ} / \mathrm{bit} / \mathrm{m}^{2}$ \\
\hline 5 & Transmission power $\left(E_{T X}\right)$ & $0.744 \mathrm{~nJ} / \mathrm{bit} / \mathrm{m}^{2}$ \\
\hline 6 & Idle power & $0.05 \mathrm{~nJ} / \mathrm{bit} / \mathrm{m}^{2}$ \\
\hline 7 & Sense power & $0.0175 \mathrm{~nJ} / \mathrm{bit}^{2} \mathrm{~m}^{2}$ \\
\hline
\end{tabular}

\section{Results and discussion}

\subsection{Simulation procedure}

The proposed model for optimal CHS in WSN using LU-GWO was executed in NS-2 tool, and the corresponding outcomes were attained. Table 1, shows the various parameters and the corresponding values in modeling CHS in WSN. The implemented CHS in WSN was compared over other traditional methods namely, LA [18], GWO [19], GA [20] Particle Swarm Optimization (PSO) [21] and Artificial Bee Colony (ABC) [22] the corresponding outcomes were obtained. Further, the experimentation was carried out in terms of alive nodes and normalized energy for 50 nodes and 100 nodes for a distance of $1000 \times 1000$ and $2000 \times 2000$. Further, the analysis was done by varying the population size of the adopted model. The simulation result of the suggested model in terms of 50 nodes and 100 nodes for a distance of $1000 \times 1000$ and $2000 \times 2000$ is given in Fig. 2.

\subsection{Analysis on alive nodes}

The performance analysis of the proposed optimal CHS in WSN system for the number of alive nodes using LU-GWO method is given by Fig. 3 . Here, with the increase of the number of rounds, the number of alive nodes gets reduced, however, the proposed model is found to exhibit number of alive nodes even at the $100^{\text {th }}$ round when compared over the existing models. From Fig. 3 (a), the proposed scheme for 50 nodes with $1000 \times 1000$ distance is $2.7 \%, 2.7 \%, 5.56 \%, 5.56 \%$, and $5.56 \%$ better than GWO, LA, GA, PSO and ABC algorithms at 20th round. In addition, from Fig. 3 (b), the implemented LU-GWO system for 50 nodes with $2000 \times 2000$ distance area is $22.22 \%, 26.92 \%$, $17.86 \%, 43.48 \%$ and $26.92 \%$ superior to GWO, LA, GA, PSO and ABC algorithms at $40^{\text {th }}$ round with respect to the number of alive nodes. From Fig. 3 (c), at 20th round, the adopted method for 100 nodes and $1000 \times 1000$ distance is $2.99 \%, 2.99 \%, 7.81 \%$, and $4.55 \%$ better than GWO, LA, GA and ABC algorithms. Also, from Fig. 3 (d), the suggested LUGWO system for 100 nodes and $2000 \times 2000$ distance is $18.52 \%, 6.67 \%, 23.07 \%, 39.13 \%$ and $60 \%$ superior to GWO, LA, GA, PSO and ABC algorithms at 100th round with respect to the number of alive nodes. Therefore, the improvements of the adopted LU-GWO technique for optimal CHS in WSN have been substantiated successfully.

\subsection{Analysis on normalized network energy}

The analysis of the proposed optimal CHS in WSN system for determining the normalized network energy using LU-GWO technique is specified by Fig. 4. From the obtained graphs, it is known that the normalized network energy gets minimized with an increase in the number of rounds. 


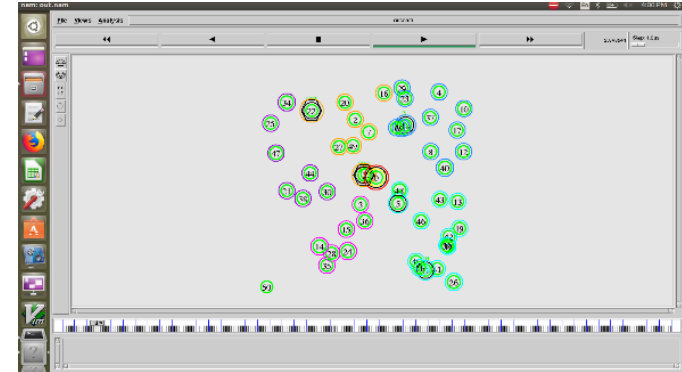

(a)

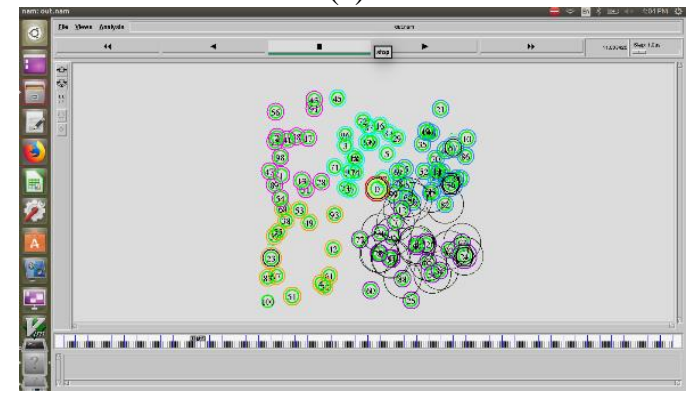

(c)

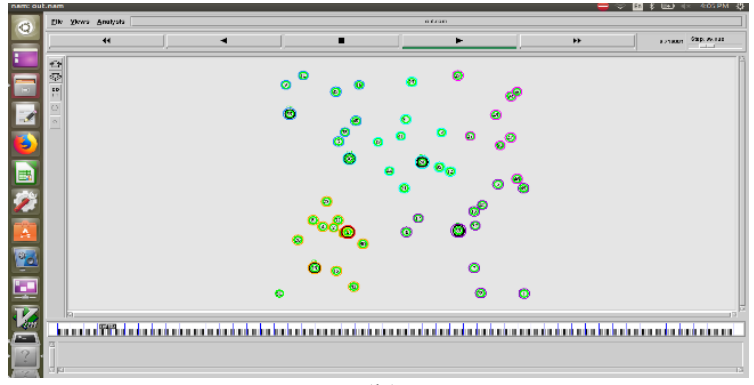

(b)

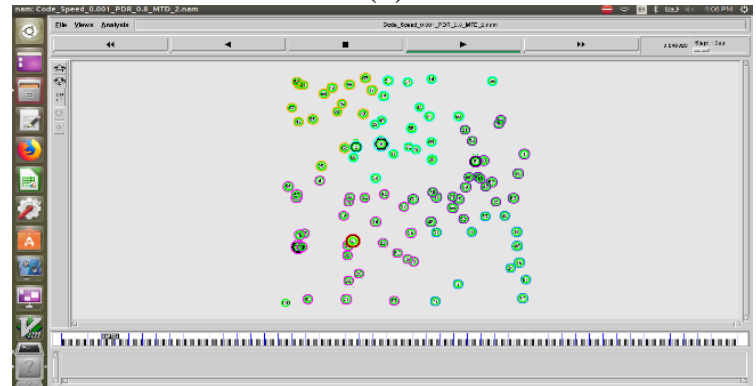

(d)

Figure. 2 Simulation results of the proposed CH selection model: (a) 50 nodes with $1000 \times 1000$ distance, (b) 50 nodes with $2000 \times 2000$ distance, (c) 100 nodes with $1000 \times 1000$ distance, and (d) 100 nodes with $2000 \times 2000$ distance

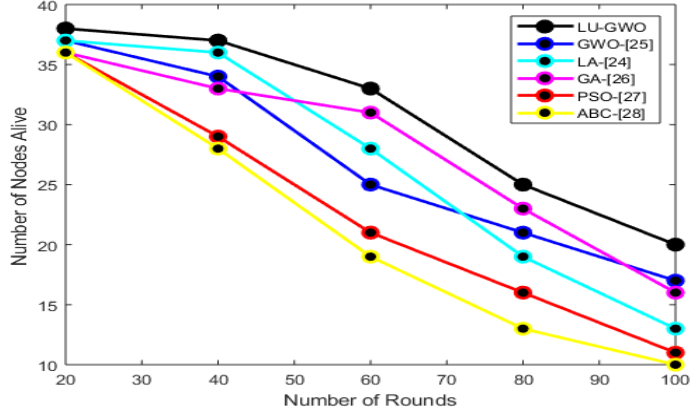

(a)

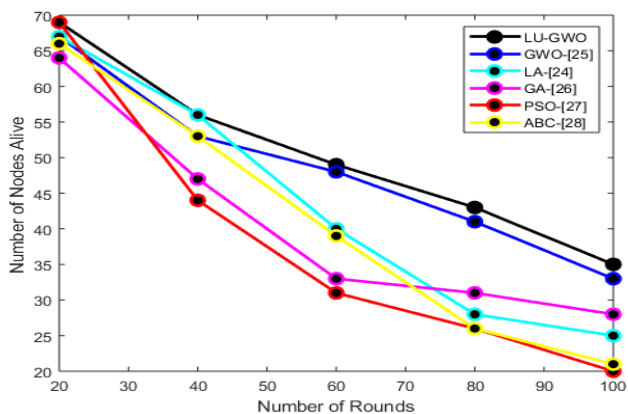

(c)

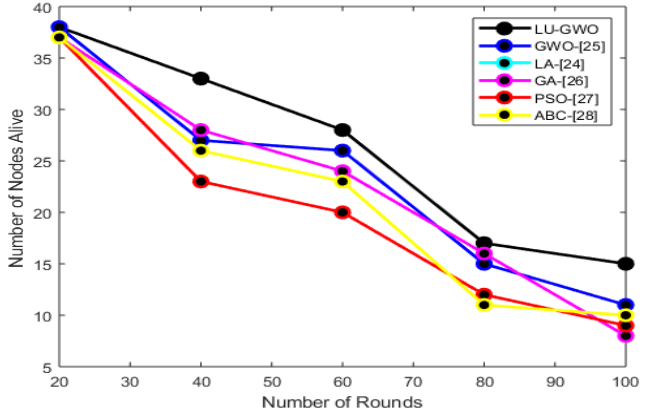

(b)

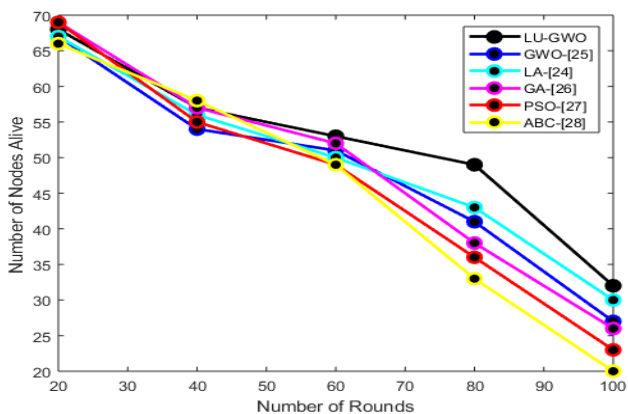

(d)

Figure. 3 Analysis on proposed and conventional models on number of alive nodes with respect to number of rounds for: (a) 50 nodes and 1000×1000 distance, (b) 50 nodes and 2000×2000 distance, (c) 100 nodes and 1000×1000 distance, and (d) 100 nodes and $2000 \times 2000$ distance

Anyhow, the presented model reveals with high network energy when evaluated over the other conventional schemes even at the 100th round. From Fig. 4 (a), the presented system for 50 nodes and $1000 \times 1000$ distance is $12.3 \%, 4.14 \%, 6.13 \%$,
$23.63 \%$, and $30.16 \%$ better than GWO, LA, GA, PSO and $\mathrm{ABC}$ algorithms at $20^{\text {th }}$ round with high normalized network energy. Moreover, from Fig. 4 (b), the adopted LU-GWO scheme for 50 nodes and 


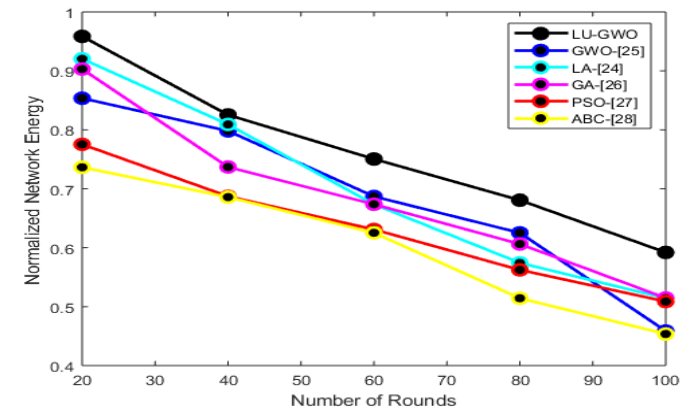

(a)

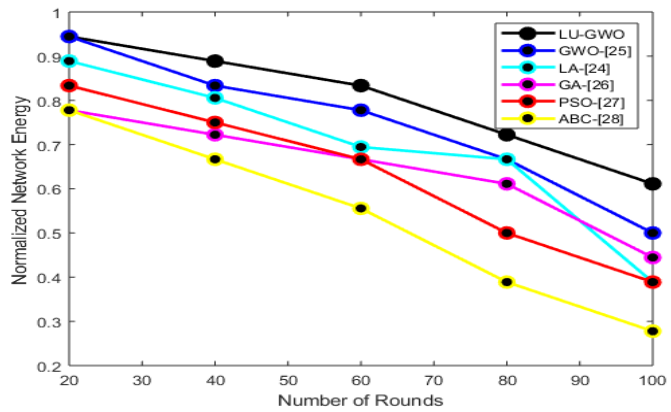

(c)

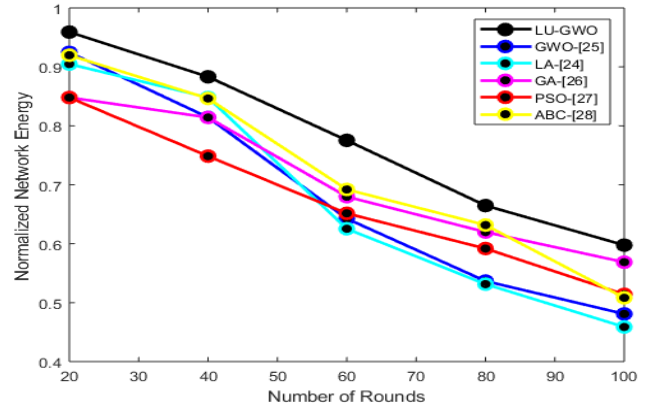

(b)

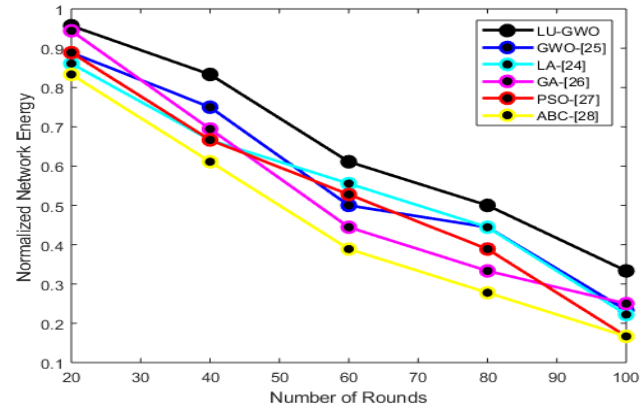

(d)

Figure. 4 Analysis on proposed and conventional models on sustaining the normalized energy of WSN with respect to number of rounds for: (a) 50 nodes and $1000 \times 1000$ distance, (b) 50 nodes and 2000×2000 distance, (c) 100 nodes and $1000 \times 1000$ distance, and (d) 100 nodes and $2000 \times 2000$ distance

$2000 \times 2000$ distance is $12.03 \%, 5.71 \%, 5.88 \%$, $10.48 \%$ and $4.08 \%$ superior to GWO, LA, GA, PSO and $\mathrm{ABC}$ algorithms at $60^{\text {th }}$ round with respect to the network energy. From Fig. 4 (c), at 100th round, the implemented method for 100 nodes and $1000 \times 1000$ distance is $22.22 \%, 57.14 \%, 37.51 \%$, $57.14 \%$, and $54.54 \%$ better than GWO, LA, GA, $\mathrm{PSO}$ and $\mathrm{ABC}$ algorithms with increased normalized network energy. In addition, from Fig. 4 (d), the proposed LU-GWO scheme for 100 nodes and $2000 \times 2000$ distance is $12.51 \%, 12.51 \%, 50 \%$, $28.57 \%$ and $79.99 \%$ superior to GWO, LA, GA, PSO and ABC algorithms at $80^{\text {th }}$ round. Thus, the superiority of the presented model has been proved over other conventional models with high normalized energy.

\section{Conclusion}

This paper has presented a novel technique for energy-aware optimal CHS by hierarchical routing in WSN using the hybrid optimization technique. The major goal was to optimally select the $\mathrm{CH}$ by concerning on the stabilization of energy, minimization of distance among nodes and minimization of delay during data transmission. Here, by means of the above-mentioned measures, a non-linear objective function was framed, which intent to optimize the $\mathrm{CH}$ in such a way that the network lifetime has to be higher. For attaining optimal CHS, the concept of LU-GWO was introduced that incorporates the concept of LA in the GWO model. Thus, optimal CHS could be efficiently determined by the adopted model. From the alive node analysis, the proposed scheme for 50 nodes and $1000 \times 1000$ distance was $2.7 \%, 2.7 \%$, $5.56 \%, 5.56 \%$, and $5.56 \%$ better than GWO, LA, $\mathrm{GA}$, PSO and $\mathrm{ABC}$ algorithms at 20th round. On considering the normalized network energy, the proposed LU-GWO scheme for 100 nodes and $2000 \times 2000$ distance was $12.51 \%, 12.51 \%, 50 \%$, $28.57 \%$ and $79.99 \%$ superior to GWO, LA, GA, $\mathrm{PSO}$ and $\mathrm{ABC}$ algorithms at 80th round. Thus, it can be known that the proposed LU-GWO methodology outperforms better than the other traditional algorithms. The future extension of this work is aimed at developing a multi-objective optimization framework for better lifetime of network based on the advanced hybrid optimization model.

\section{References}

[1] A. Mazinani, S. M. Mazinani, and M. Mirzaie, "FMCR-CT: An energy-efficient fuzzy multi cluster-based routing with a constant threshold in wireless sensor network", Alexandria Engg. Journal, Vol.58, No.1, pp.127-141, 2019. 
[2] T. Wang, G. Zhang, X. Yang, and A. Vajdi, "Genetic algorithm for energy-efficient clustering and routing in wireless sensor networks", Journal of Systems and Software, Vol. 146, pp. 196-214, 2018.

[3] A. S. Toor and A. K. Jain, "Energy Aware Cluster Based Multi-hop Energy Efficient Routing Protocol using Multiple Mobile Nodes (MEACBM) in Wireless Sensor Networks", AEU-InternationalJournal of Electronics and Communications, Vol. 102, pp. 41-53,2019.

[4] K. Muthukumaran, K. Chitra, and C. Selvakumar, "An energy efficient clustering scheme using multilevel routing for wireless sensor network", Computers \& Electrical Engineering, Vol. 69, pp. 642-652, 2018.

[5] M. Sabet and H. R. Naji, "A decentralized energy efficient hierarchical cluster-based routing algorithm for wireless sensor networks", $A E U$ - International Journal of Electronics and Comm., Vol. 69, No. 5, pp. 790-799, 2015.

[6] J. An, L. Qi, X. Gui, and Z. Peng, "Joint design of hierarchical topology control and routing design for heterogeneous wireless sensor networks", Computer Standards \& Interfaces, Vol. 51, Issue. C, pp. 63-70, 2017.

[7] W. Ke, O. Yangrui, J. Hong, Z. Heli, and L. Xi, "Energy aware hierarchical cluster-based routing protocol for WSNs", The Journal of China Universities of Posts and Telecommuni., Vol. 23, No. 4, pp. 46-52, 2016.

[8] N. Sabor, M.S. Ahmed, M. Zahhad, and S. Sasakil, "ARBIC: An Adjustable Range Based Immune hierarchy Clustering protocol supporting mobility of Wireless Sensor Networks", Pervasive and Mobile Computing, Vol. 43, pp. 27-48, 2018.

[9] T. Gaber, S. Abdelwahab, M. Elhoseny, and A. E. Hassanien, "Trust-based secure clustering in WSN-based intelligent transportation systems", Computer Networks, Vol. 146, pp. 151-158, 2018.

[10] V. Kumar, V. Kumar, D.N. Sandeep, S. Yadav, and S. Tiwari, "Multi-hop Communication based optimal clustering in hexagon and voronoi cell structured WSNs", $A E U$ InternationalJournal of Electronics and Communications, Vol. 93, pp. 305-316, 2018.

[11] A. Kumar, P. Pahwa, D. Virmani, and S. Swami, "Dynamic CH Selection Using Fuzzy Logic on Cloud in Wireless Sensor Networks", Procedia Computer Science, Vol. 48, pp. 497502, 2015.

[12] V. Geetha, P. V. Kallapur, and S. Tellajeera, "Clustering in Wireless Sensor Networks:
Performance Comparison of LEACH \& LEACH-C Protocols Using NS2", Procedia Technology, Vol. 4, pp. 163-170, 2012.

[13] M. Masdari, S.M. Bazarchi, and M. Bidaki, "Analysis of Secure LEACH-Based Clustering Protocols in Wireless Sensor Networks", Journal of Network and Computer Appli., Vol. 36, No. 4, pp. 1243-1260, 2013.

[14] R.K.Yadav and R.P.Mahapatra, "Descendant of LEACH Based Routing Protocols in Wireless Sensor Networks: A Survey," Procedia Computer Science, Vol. 57, pp.1005-1014, 2015.

[15] O. Rehman, N. Javaid, B. Manzoor, A. Hafeez, and M. Ishfaq, "Energy Consumption Rate based Stable Election Protocol for WSNs", Procedia Computer Science, Vol. 19, pp. 932937, 2013.

[16] R. Mahakud, S. Rath, M. Samantaray, S. Sinha, and A. Kumari, "Energy Management in Wireless Sensor Network Using PEGASIS", Computer Science, Vol. 92, pp. 207-212, 2016.

[17] A. Jedidi, "Workload Cluster Balance Algorithm to Improve Wireless Sensor Network Performance", International Journal of Communication Networks and Information Security, Vol. 11, No. 1, pp.105-111, 2019.

[18] B. R. Rajakumar, "The Lion's Algorithm: A New Nature-Inspired Search Algorithm", Procedia Technology, Vol. 6, pp. 126-135, 2012.

[19] S. Mirjalili, S. M. Mirjalili, and A. Lewis, "Grey Wolf Optimizer", Advances in Engineering Software, Vol. 69, pp.46-61, 2014.

[20] J. McCall, "Genetic algorithms for modelling and optimisation", Journal of Computational and Applied Mathematics, Vol. 184, No. 1, pp. 205-222, 2005.

[21] J. Zhang and P. Xia, "An improved PSO algorithm for parameter identification of nonlinear dynamic hysteretic models", Journal of Sound and Vibration, Vol. 389, pp. 153-167, 2017.

[22] M. S. Kiran and O. Findik, "A directed artificial bee colony algorithm", Applied Soft Computing, Vol. 26, pp. 454-462, 2015. 